then be close to the lines and the field hospitals need not be far off. I have sometimes located them less than 300 yards from the front, and even ambulances, conspicuous marks as they are, can sometimes be driven along the hollows, taking the wounded directly from the first aid stations.

Surgeon Fischer, of the Austrian army, thinks the number of wounded will be greatly increased. It seems to me that this popular idea is erroneous. Most of the fighting will be at greater distances than it was formerly, and the new guns are no more accurate than the old ones. Beside, experience shows that even well disciplined men can rarely be made to stick to their work after 30 per cent. of their number are hit. This old rule will be as true in the future as in the past, and no change in weapons will prevent men from shrinking from danger when it exceeds their fortitude.

Fischer also thinks the mortality of the wounds will be doubled, but Melville dissents from that conclusion.

The facts point in two directions. A few wounds will perhaps bleed more than the old ones, on account of swift shots cutting more like a knife than slow ones, but we must remember that the injuries will be inflicted at greater distances, when the bullets have largely lost their velocity.

Close shots will tear and shatter more in consequence of what is called the "explosive effect," bat these only occur at near range. Most of the injuries will take place at greater distances, and the bullets being very small, the wounds will on the average be less fatal from shock, and less open to septic infection. Many will heal by first intention.

On the whole, I think that no more men will be hit than formerly, and that of those wounded more will recover.

Finally, all the utensils and everything about the field hospitals must be made as simple and portable as possible, so that they can be thrown into the wagon and rushed forward, at a moment's notice, to where the wounded lie, as soon as the fighting admits of the advance. In this way the men can be taken care of without waiting for the slow, interminable and exhausting work of litter bearers.

2520 Prairie Ave., Chicago.

\section{E. WERTHEIM'S NEW METHOD OF RE- STORING RETRODEVIATIONS OF THE UTERUS THROUGH THE VAGINA.}

BY A. J. HOSMER, M.D. ASHLAND, WIS.

The operation consists in shortening the round ligament through the vagina. The technic of the operation in uncomplicated cases is as follows: After the necessary antiseptic precautions, the patient is placed in the exaggerated lithotomy position. (A curettement is performed, when indicated.) The limbs are supported, retractors used, and the cervix uteri is seized with bullet forceps and drawn down, as in ve.ginal hysterectomy. With a scalpel, a transverse incision about one and one-half inches long is made at the anterior utero-vaginal junction, down to the uterus. Now with the index finger or with blunt pointed scissors, the uterus is separated from the bladder. The vesico-uterine space (anterior cul-desac), or rather the peritoneum lining it, is reached with ease. The peritoneum is seized with two anatomic forceps and opened between them with the scissors. The incision is then extended transversely about an inch each way. Now while still holding to the ante-

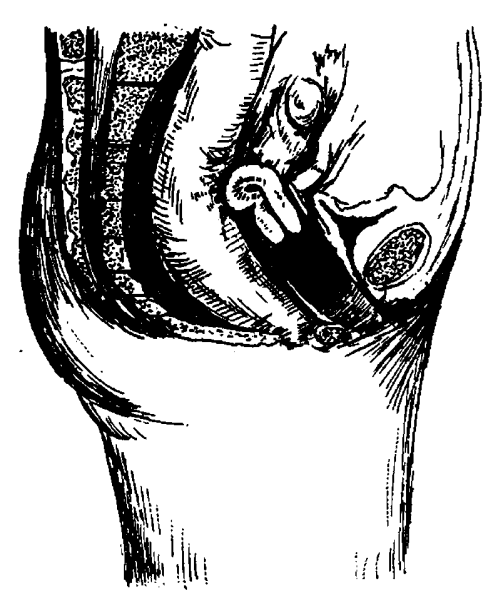

FigURE 1. rior peritoneal flap with one of the forceps, a needle, with medium sized silk, is passed through it and attaches it to the cut edge of the anterior vaginal wall.

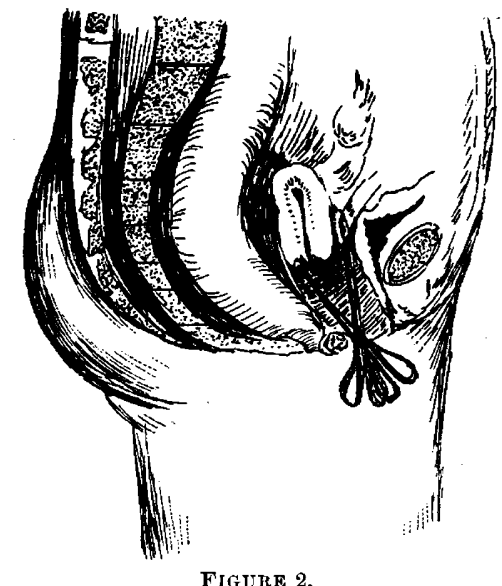

Two other stitches are used attaching peritoneal flap to the cut edge of the vagina, thus entirely covering the raw surface of the bladder. Easily now with the

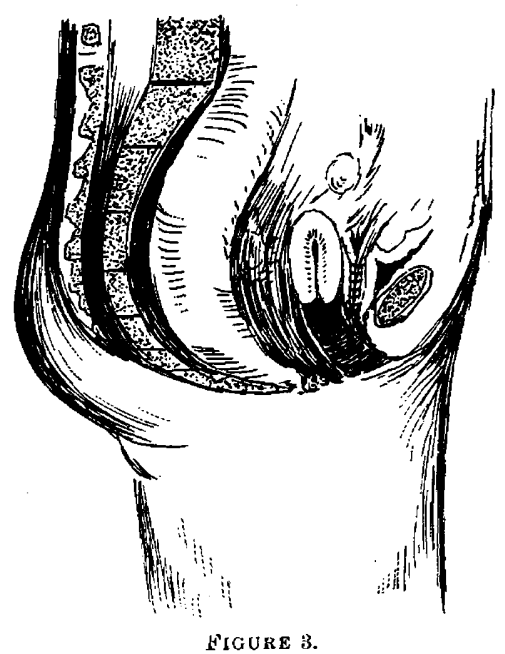

fingers one can reach the body of the uterus and ascertain its condition as well as the state of the Fallopian tubes and ovaries. When necessary, loosen adhesions, replace ovaries, remove small subserous myomas, etc. 
Next, unfasten the bullet forceps from the cervix, allowing it to go back as you roll the body of the uterus forward into the vagina or at least into plain view. If there are no adhesions, the uterus comes forward without force. If there are adhesions they are easily reached with the fingers and separated.

The round ligament is the first cord in sight and can be seen, examined and differentiated from ligamentum proprium and Fallopian tube. The ligament is seized from one to two inches from the horn of the uterus, with bullet forceps and drawn out, thus necessarily doubliny it upon itself. With medium sized silk, its approximating doubled surfaces are sewed together. By thus folding it upon itself by the use of three or four stitches, the round ligament can be shortened from six to nine centimeters.

The other round ligament is treated in like manner, and thus the uterus is held up and anteverted. The stitches holding the peritoneum to vagina are now cut and the two peritoneal surfaces re-united. The transverse incision at the utero-vaginal junction is then sewed vertically thus lengthening the anterior vaginal wall, and shoving the cervix back.

A little gauze is placed in the vagina and the operation is finished, in most cases without having tied a blood vessel, and unless the wound has been infected, without danger.

There is no danger of injuring the ureters, if the. operation is done as described above, as they are shoved out of the way. In cases where there is a relaxed vagina and an anterior colporrhaphy is needed, instead of making the transverse incision, an elliptic piece can be removed from the anterior vaginal wall, and the bladder separated from the uterus directly under the eye. The round ligaments are treated as in the first case. In sewing up the vaginal wall you have, of course, done an anterior colporrhaphy.

This operation was first made by $\mathbf{E}$. Wertheim of Vienna, in January, 1896, and his first publication of the same appeared in the Centralblatt für Gynäkologie in February, 1896, since which time it has been performed about twenty-five times and with perfect results.

Its advantages over the Alexander-Adams operation are: 1 , it leaves no visible 'scar; 2 , the operation is easier to perform, the ligaments being larger at this point and easy to find; 3 , the transverse cut in the vagina, sewed vertically, assists in anteverting the uterus; 4 , it allows perfect access to the pelvic viscera, thus permitting of minor repairing at the same sitting, when indicated; 5, the operation is less dangerous.

Its advantages over any method of anterior fixation which will prevent the free growing of a gravid uterus are very apparent. The bladder is left in its normal position. A subsequent pregnancy will not be hindered, as the shortened ligaments can stretch as in the normal state. That every antefixation which will prevent the free growing of a gravid uterus is likely to be the source of danger, is well illustrated in Miländer's report (Zeitschrift für Geburtshülfe und Gynäkologie. Bd. xxxiii, Hftt. 3). In fifty-four cases of full term labor after ventrofixation, eleven were operative, four forceps, two Cæsarean sections, four turning and one extraction.

The uterus is not only anteverted, but it is held up, as one can very easily prove by doing the operation upon the cadaver, and the organ is not antefixed, but held in its normal position by its normal attachments.
This operation I have witnessed frequently in Schauta's operating room, and the ease and rapidity with which it is done, beside its many advantages over other known methods, is my excuse for sending this report.

For the past year, I have not had access to any medical journal except the Journal of THE AMERICan Medical Association, hence if this method of E. Wertheim's has been reported in an American medical journal, I am ignorant of the fact.

\section{A NEW APPARATUS FOR FRACTURE OF THE CLAVICLE.}

BY EVAN O'NEILT KANE, M.D. KANE, PENN.

The treatment for fracture of the clavicle is almost uniformly unsuccessful. Were it not that the resulting rounded shoulder, narrowed chest and bone deformity do not materially injure the patient, the best surgeons would be continually mulcted in malpractice suits.

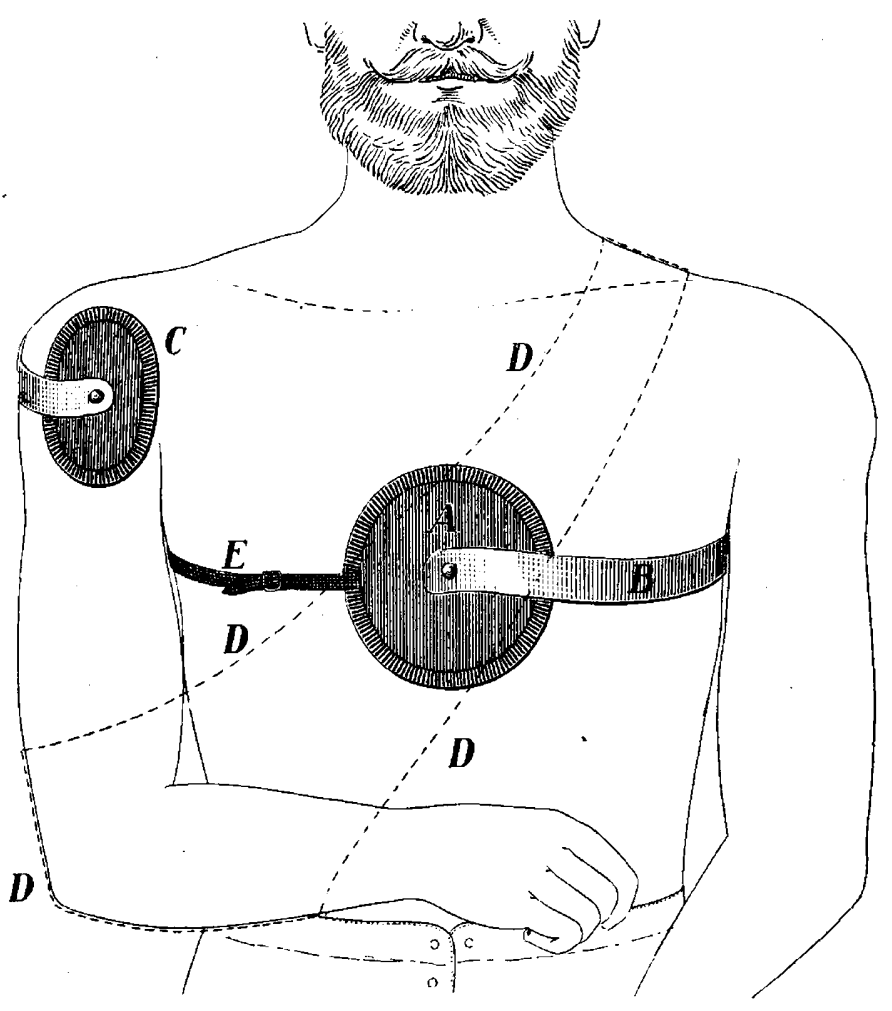

Front. A, chest pad; B, spring on sound side; C, shoulder pad and spring on injured side; $D$, dotted lines indicating ordinary sling; $E$, elastic strap and buckle.

It is true that if the patient can be placed for three or four weeks upon his back; a narrow cushion below and a shot.bag above, a perfect result may be obtained; but few can bear with so irksome a treatment for so comparatively trivial an injury. No other methods of treatment produce perfect results; and all are painful or even prejudicial to the patient's health. Many of these apparatus are now discarded as cumbrous, complicated and unsatisfactory. the pad in the axilla sooner or later exerts so much pressure upon the nerves and vessels as to require its removal. Bandaging the arm across the chest, rarely attains more than fixation and the prevention of falling of the shoulder, while it so cramps the arm and constricts 